\title{
Diacronie
}

Studi di Storia Contemporanea

$N^{\circ} 17,1 \mid 2014$

Periferie. Cultura, economia, politica

\section{Nota introduttiva n. 17 - marzo 2014}

Jacopo Bassi, Luca Bufarale e Matteo Tomasoni

\section{(2) OpenEdition}

\section{Journals}

\section{Edizione digitale}

URL: http://journals.openedition.org/diacronie/981

DOI: 10.4000/diacronie.981

ISSN: 2038-0925

\section{Editore}

Association culturelle Diacronie

\section{Notizia bibliografica digitale}

Jacopo Bassi, Luca Bufarale e Matteo Tomasoni, « Nota introduttiva n. 17 - marzo 2014 », Diacronie [Online], N 17, 1 | 2014, Messo online il 01 mars 2014, consultato il 24 septembre 2020. URL : http:// journals.openedition.org/diacronie/981 ; DOI : https://doi.org/10.4000/diacronie.981

Questo documento è stato generato automaticamente il 24 settembre 2020.

Creative Commons License 


\title{
Nota introduttiva n. 17 - marzo 2014
}

\author{
Jacopo Bassi, Luca Bufarale e Matteo Tomasoni
}

1 Il numero 17 di Diacronie si articola intorno al concetto di periferia. Il termine - complesso e il cui significato spazia dall'ambito urbanistico a quello geografico, passando per l'economia e la politica - riunisce dodici articoli, differenti per approccio, tematica affrontata e strumenti d'indagine adottati.

Il lettore si troverà di fronte a un

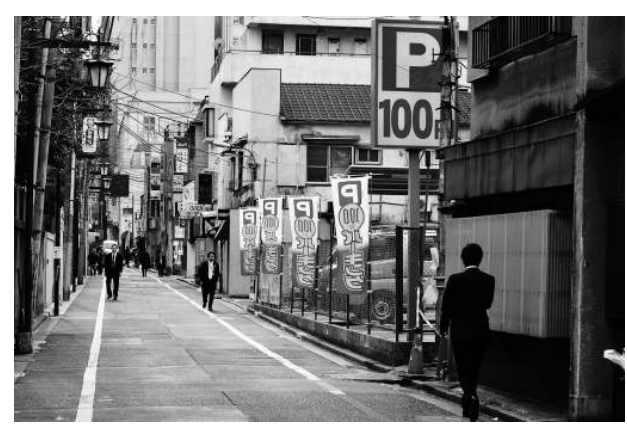
interrogativo che sorge dalla suddivisione in sezioni che è stata qui proposta: ha ancora senso adottare un approccio rigidamente "diffusionista» ${ }^{1}$ che individui nella cultura, nell'economia o nella politica il cardine intorno a cui ruota la dialettica centro-periferia?

3 La risposta - a dispetto della suddivisione in sezioni qui proposta - riteniamo che sia negativa. Come si noterà leggendo i saggi, l'analisi dei casi di studio fa emergere una necessaria contaminazione nel metodo d'analisi impiegato dagli autori. L'interpretazione proposta non è mai, in nessun caso, monoliticamente rivolta ad uno solo degli aspetti sopra elencati. L'aspetto preponderante è l'individuazione dell'origine dei cambiamenti che portano ad una ridiscussione dei rapporti fra centro e periferia; per usare le parole di Eisenstadt:

[...] gli orientamenti rivoluzionari alla radice di moltissimi balzi verso la modernità, avevano mirato ad una trasformazione completa della natura e del contenuto del centro dell'ordine sociale e culturale, delle norme che regolano la partecipazione e l'accesso al medesimo, e dei rapporti intercorrenti tra il centro e la periferia.

Per quanto concerne il contenuto di tali centri, la principale trasformazione verificatasi in concomitanza all'ingresso della modernità è stata la loro sempre più netta secolarizzazione, l'«apertura» del loro stesso contenuto: contenuti e simboli, 
cioè, cominciarono a non essere più accettati come dei dati di fatto, mentre si diffondeva l'idea della possibilità di un loro riesame. ${ }^{2}$

4 Ed è proprio a partire dalla messa in discussione dei rapporti centro periferia che si articolano i saggi qui presentati; la ridefinizione di queste relazioni non porta necessariamente in tutte le occasioni ad un miglioramento delle condizioni per le periferie, ma è indice del raggiungimento di un punto di rottura nelle relazioni preesistenti.

5 La prima sezione è consacrata al tema del rapporto tra la diffusione dei modelli culturali nelle periferie "geografiche" e la rielaborazione di questi stessi.

Muriel Marchal, con il suo saggio su Atlantica di Olaus Rudbeck prende in esame il processo di consolidamento della Svezia tanto come potenza regionale (nel Baltico) quanto come internazionale (in Europa) nel XVII secolo; lo fa prendendo in considerazione la produzione culturale del paese scandinavo. L'attenzione è rivolta ad Atlantica, opera di Olaus Rudbeck, scienziato e scrittore: attraverso questo trattato l'autore desiderava comprovare come la Svezia fosse, in realtà, il crogiolo della civiltà classica. In questo caso la rielaborazione di un corpus letterario e mitologico - divenuto patrimonio comune europeo - era finalizzata all'elaborazione di una nuova storia nazionale che potesse essere in grado di nobilitare la monarchia svedese; è opportuno tenere in considerazione anche lo status di inferiorità di cui godeva, all'epoca, lo svedese come lingua letteraria ${ }^{3}$. L'opera di Rudbeck avrebbe poi dato l'avvio al movimento goticista.

7 Marie-Claude Lepeltier, con il suo articolo - che appare qui in traduzione italiana per gentile concessione della rivista Études corses - affronta il tema dell'autonomismo corso passando in rassegna le vignette apparse sul giornale «A Muvra». Fondato nel 1920 - e parzialmente in lingua corsa - divenne l'organo del Partitu corsu autonomista. Le vignette satiriche metto in evidenza i motivi del malessere corso e l'insofferenza nei confronti del “centro" francese, vissuto - sulle pagine di «A Muvra» - al contempo come sfruttatore, colonizzatore e censore. Il regionalismo degli anni Venti, pur non mettendo mai in discussione il legame fra l'isola e la Francia ${ }^{4}$ conobbe una rapida radicalizzazione sfociando nell'irredentismo e compromettendosi, nel corso degli anni Trenta, con il regime fascista.

8 Due articoli - nel solco di un consolidato progetto progetto di scambio e collaborazione con il gruppo di studiosi di GET, Grupo de Estudio do Tempo presente - gettano uno sguardo sull'influenza di alcuni modelli culturali nella società brasiliana.

Dilton Cândido S. Maynard si sofferma sulla produzione letteraria di Mário de Andrade, poeta e scrittore fra i fondatori del modernismo brasiliano. Proprio a partire dai suoi scritti il saggio proposto mette in rilievo la figura di Delmiro Gouveia quale contraltare a Macunaíma, il più celebre dei personaggi di De Andrade. Se Macunaíma è l'anti-eroe per eccellenza - odioso, dedito ai vizi e infido -, Delmiro Gouveia viene proposto come modello di un Brasile diverso, dedito al progresso, al lavoro ed eticamente inappuntabile. La figura storica di Gouveia - imprenditore brasiliano, "colonnello dei colonnelli" - diviene, attraverso l'antologia di brani presi in esame da Santos Maynard, dà luogo anch'essa a un personaggio simil-letterario, quasi il topos (in maniera probabilmente persino eccessiva in considerazione delle ombre che comunque gravano su Gouveia) del Brasile dei primi decenni del XX secolo, che ambisce a modernizzare se stesso inseguendo l'idea di progresso. 
10 Andreza Cruz Santos Maynard analizza l'avvento massiccio della produzione cinematografica statunitense nelle sale di proiezione brasiliane. Frutto del varo della politica roosveltiana di "buon vicinato", l'afflusso di pellicole nordamericane sul mercato sudamericano - e brasiliano nel caso specifico -, contribuì anche economicamente al successo delle case di produzione americane. Il mutamento dell'immagine del vicino latinoamericano, che originò un vero e proprio filone cinematografico $^{5}$, al quale prese parte anche la Disney (Saludos amigos, The tree caballeros), si accompagnò così all'allineamento del Brasile al campo alleato (il Brasile dichiarò guerra all'Italia e alla Germania il 22 agosto 1942). Si venne così a costituire un rapporto basato anche sulla trasmissione di prodotti culturali fra il centro statunitense e la periferia latinamericana che sarebbe, anche se solo parzialmente, entrato in crisi solo con l'inizio della Guerra fredda.

11 La sezione dedicata all'approccio economico al concetto di periferia si apre con il saggio di Milena Fernandes de Oliveira. Lo studio qui proposto mette in luce come le idee economiche dell'illuminismo portoghese, importate in un paese ancora profondamente legato all'ideologia economica d'Antico Regime dall'estrangeirado (cioè da quegli studiosi formatisi all'estero e latori di ideologie provenienti da altri paesi), abbiano posto le basi per la nascita di due nuovi nuclei statali: il Portogallo e i Brasile. L'idea di nazione, nel caso luso-brasiliano, sarebbe dunque nata a partire dall'economia politica e dall'idea di mercato.

12 Alessandro De Luca propone un saggio sulla marginalità sociale nel Dipartimento del Taro durante l'epoca napoleonica, toccando così al contempo aspetti economici, sociali e geografici. Proprio durante questo cruciale tornante storico, per effetto dell'incameramento statale delle proprietà ecclesiastiche abbinato ad una nuova concezione statale, si posero le basi per un nuovo approccio nei confronti dei marginali. La dominazione francese con la sua efficenza amministrativa fornisce una quantità di dati preziosa per il lavoro storiografico.

13 Francesca Sanna prende in esame un caso di rapporto centro-periferia nella gestione delle risorse territoriali: il declino, avviatosi nel secondo dopoguerra, dell'industria mineraria in Sardegna in favore del settore petrolchimico. Per effetto dei finanziamenti pubblici per la riconversione e la verticalizzazione (spiccano in questo i Piani di Rinascita) l'interesse generale si volse verso uno sviluppo basato sull'industria petrolifera. Fu così realizzato un settore industriale che non aveva legami con il territorio, mentre le miniere - gestite in maniera inefficiente - finirono per esaurirsi o per essere chiuse.

14 Gli articoli della terza sezione sono accomunati, pur nella diversità delle tematiche, da un approccio alla storia politica che privilegia i momenti di crisi identitaria e di reciproca influenza che hanno caratterizzato le vicende di partiti e di movimenti dall'Italia all'Argentina, dalla Spagna alla Turchia. Sono proprio queste aree ad essere oggetto dei cinque saggi della sezione in cui la perifericità - o meglio, la semiperifericità ${ }^{6}$ - è una condizione transitiva rispetto all'assetto politico nazionale e internazionale. Si spazia così dal rapporto ambivalente tra sindacalismo rivoluzionario e fascismo, alle influenze della guerra civile spagnola nella lotta politica argentina, dalla crisi del Partito comunista italiano nella seconda metà degli anni Ottanta a quella dell'Unione di centro spagnola all'inizio dello stesso decennio, sino all'ascesa, sotto vari nomi, del partito islamico di Erbakan nella Turchia laica. In almeno due casi - l'articolo 
sull'Argentina e quello sull'UCD spagnola - gli autori hanno scelto di occuparsi di una problematica più ampia a partire da casi locali.

Apre la sezione il saggio di Marco Masulli focalizzato su un tipico movimento "di confine" della storia politica italiana: il sindacalismo rivoluzionario. Nato "a sinistra" come contestazione delle tendenze riformistiche e gradualiste del socialismo italiano, il movimento di De Ambris, Corridoni e Olivetti contribuisce ad influenzare il fascismo "della prima ora", quello che, mettendo insieme fermenti nazionalistici, istanze sociali e una generale ripulsa nei confronti dei partiti dell'anteguerra, possiede, per usare una celebre espressione di Salvatorelli, un "volto di sfinge". Mettendo in discussione l'interpretazione che ritiene tale convergenza quasi scontata data la comune matrice antiparlamentaristica e volontaristica, l'autore individua diversi momenti dell'incontro tra sindacalismo rivoluzionario e fascismo, soffermandosi in particolare sulla lotta contro il riformismo turatiano, sulla posizione interventista durante il primo conflitto mondiale e sulla concezione del corporativismo. Nell'idea di una società di "liberi produttori" risiede infatti il maggiore lascito del sindacalismo rivoluzionario al multiforme bagaglio culturale del fascismo ma anche il più aspro terreno di scontro, una volta che molti sindacalisti rivoluzionari si avvedono della sostanziale subalternità del nuovo sindacato fascista al partito-stato e alle richieste degli industriali.

L'articolo di Dario Diez Miguel analizza la decomposizione del partito centrista UCD, guidato da Adolfo Suarez nel contesto della transizione spagnola alla democrazia nei primi anni Ottanta. Erede dei settori "riformisti" del tardo regime franchista, l'Unión de Centro Democrático ingloba anche esponenti cattolici, liberali e socialdemocratici. La progressiva decomposizione di questa forza politica viene indagata attraverso un caso-studio costituito dalla provincia di Zamora (nella Castiglia-Leon). Utilizzando diverse fonti d'archivio, molte delle quali inedite, Díez propone quindi un valido approfondimento, attraverso il quale siamo in grado di comprendere meglio l'involuzione politica della UCD tra la fine degli anni Settanta e l'inizio degli Ottanta; processo che anche nel caso di Zamora qui preso in esame, caratterizzò una parte del gruppo che finì per seguire il suo massimo dirigente, Suárez, nella nuova avventura politica rappresentata dal Centro Democrático y Social (CDS). L'adozione di una prospettiva locale permette all'autore di far risaltare l'importanza dello scontro tra centro e periferia (laddove la periferia è rappresentata, a differenza che in altri contesti, da un territorio senza particolari tendenze autonomistiche) e delle lotte di potere interne, caratteristiche della crisi del partito, in una prospettiva che al lettore italiano può ricordare, per alcuni versi, il processo di decomposizione della Democrazia cristiana all'inizio degli anni Novanta.

Il saggio di Emanuela Locci ha per argomento la lenta ma costante ascesa del partito islamico in Turchia negli ultimi quarant'anni seguendo le vicende politiche di uno dei suoi leader principali, Necmettin Erbakan. Considerato, sia pure con alcune differenze, il progenitore dell'AKP del primo ministro Erdogan al governo in Turchia da quasi un decennio e recentemente fatto oggetto di aspre contestazioni, Erbakan ha promosso varie formazioni che con svariate denominazioni (Partito per l'Ordine Nazionale, Partito del Benessere Nazionale, Partito della Prosperità, Partito della Felicità) si rifanno all'Islam politico. Malgrado l'opposizione dei vertici militari, Erbakan è stato negli anni Novanta il primo premier islamista in una Turchia che, a partire dalla rivoluzione kemalista dei primi anni Venti, ha fatto della laicità dello stato uno dei suoi caratteri distintivi. L'autrice evidenzia le contraddizioni del suo movimento politico, in 
cui le prese di posizione a favore dei ceti più svantaggiati convivono con il sostegno ai processi di privatizzazione e gli stessi richiami all'islamismo si accompagnano ad una sorta di neo-nazionalismo (disposto però anche ad una convivenza con l'elemento curdo sulla base della comune identità islamica) senza che ciò comporti una rottura dei rapporti con l'Europa e gli USA. L'ascesa dell'islamismo diventa una sorta di cartina tornasole dei cambiamenti sociali e culturali della Turchia degli ultimi quarant'anni e della crisi dell'ideologia kemalista come collante della società turca.

Rebeca Camaño Semprini si occupa invece dei riflessi della guerra civile spagnola nella politica argentina della seconda metà degli anni Trenta scegliendo la provincia di Cordoba come caso studio. Facendo propria l'interpretazione di Hobsbawm del conflitto in Spagna come prima guerra ideologica internazionale per la sua capacità di mobilitare l'opinione mondiale (e non soltanto a livello puramente ideologico), l'autrice si concentra sulla visione che la stampa conservatrice e i gruppi cattolici e parafascisti danno del governo provinciale presieduto dal radicale Amadeo Sabattini. Quest'ultimo, infatti, viene accusato, per le sue politiche sociali, le sue aperture ai comunisti e le sue misure contro l'eversione di destra, di portare l'Argentina verso la stessa situazione di tensione della Spagna del 1936. La tesi del saggio è che i movimenti dell'estrema destra argentina, inizialmente alquanto minoritari, si galvanizzano e trovano una loro legittimazione presso i settori più conservatori della società argentina proprio riproponendo pedissequamente il parallelismo tra il Fronte popolare spagnolo e il governo provinciale di Sabattini e tra il pronunciamiento di Franco, necessario per salvare la Spagna dal caos, e la richiesta, da loro avanzata più volte, di un intervento del governo federale contro l'esecutivo Sabattini.

Chiude la sezione il saggio di Michelangela Di Giacomo sul Partito comunista italiano durante la segreteria di Alessandro Natta nella seconda metà degli anni Ottanta. Partito centralizzato e al tempo stesso fortemente ramificato nel territorio, con un grande bagaglio culturale e teorico alle spalle, il PCI viene attraversato da una crisi che per l'autrice è, prima ancora che politica, identitaria.

Divenuto segretario dopo la scomparsa nel 1984 di un leader carismatico come Enrico Berlinguer, Natta opera una difficile mediazione tra l'ala "migliorista" che punta al definitivo avvicinamento del $\mathrm{PCI}$ alle socialdemocrazie europee e a rapporti più stretti con i socialisti di Craxi (in quel momento a capo di un governo "pentapartito" con democristiani, socialdemocratici, repubblicani e liberali) e la "sinistra" interna, attenta a non perdere i legami con il mondo operaio e i movimenti sociali emersi nel decennio precedente. Tale mediazione viene resa possibile, secondo l'autrice, propria da quell'autorappresentazione del PCI come "soggetto-forte" in grado di superare gli equilibri politici e sociali determinatisi in Italia dal 1947-1948 e di proporre una "via italiana al socialismo" che ancora negli anni ottanta continua a prevalere nei quadri così come nei militanti di base. Il venir meno di tale autorappresentazione, specie presso gli intellettuali e i dirigenti più giovani, risulta quindi centrale per capire come il più forte partito comunista dell'Europa occidentale, arrivato con le elezioni europee del 1984 all'obiettivo del "sorpasso" della DC e capace l'anno seguente di raccogliere la sfida del governo Craxi sul referendum per la scala mobile, abbia nel volgere di pochi anni cambiato nome, simbolo e ragione sociale.

21 Un ringraziamento particolare va ad Ange Rovere e Christian Peri per l'aiuto offerto facendoci avere il materiale iconografico necessario a offrire questa versione italiana 
dell'articolo di Marie-Claude Lepeltier e alla rivista Études Corses per aver dato la propria disponibilità alla traduzione del saggio, comparso sul fascicolo numero 64 .

Un grazie a Cristina Celani per il paziente lavoro di rilettura e di correzione di bozze, svolto negli ultimi giorni del suo tirocinio, con i consueti zelo, precisione e rapidità e a Carlos Hudson per il suo prezioso lavoro di revisione e assistenza, anche in questa occasione svolto con la solita disponibilità e spirito di servizio.

Obrigado ad Anita Lucchesi che, malgrado la tesi da discutere e gli altri mille impegni, ha trovato il tempo per aiutarci traducendo l'articolo di Andreza Cruz Santos Maynard.

\section{NOTE}

1. TARROW, Sidney, Tra centro e periferia: il ruolo degli amministratori locali in Italia e in Francia, Bologna, Il Mulino, 1979.

2. EISENSTADT, Shmuel N., Civiltà comparate. Le radici storiche della modernizzazione, Napoli, Liguori, 1990, p. 46.

3. KIRBY, David, Northern Europe in the Early Modern Period. The Baltic World 1492-1772, London-New York, Longman, 1990, p. 286.

4. PELLEGRINETTI, Jean-Paul, ROVERE, Ange, La corse et la République. La vie politique de la fin du second Empire au début du XXIe siècle, Paris, Editions du Seuil, 2004, p. 245.

5. WOLL, Allen L., «Hollywood's Good Neighbor Policy», in Journal of Popular Film, 3, 4/1974, pp. 278-293.

6. ARRIGHI, Giovanni, Introduction, in ID. (ed.), Semiperipherial Development. The politics of Southern Europe in the Twentieth Century, Beverly Hills-London-New Dehli, Sage Publications, 1985, pp. 11-27.

\section{AUTORI}

\section{JACOPO BASSI}

Nel 2006 consegue la Laurea Triennale in « Storia del mondo contemporaneo » presso l'Università di Bologna sostenendo una tesi in Storia e istituzioni della Chiesa ortodossa dal titolo Tra Costantinopoli e Atene: Il passaggio delle diocesi dell'Epiro all'amministrazione della Chiesa di Grecia e la 'Praxis' del 1928, relatore il Professor Enrico Morini. Nel 2007, nel quadro del programma di scambio Erasmus, ha frequentato per un trimestre l'École Normale Supérieure (ENS) di Parigi; ha effettuato un periodo di soggiorno durante i mesi di gennaio e febbraio 2008 presso l' École Française d' Athènes, sotto il tutorato del dottor Anastassios Anastassiadis, membro del comitato scientifico di questa istituzione. Nel luglio 2008 ha discusso la Tesi Specialistica in Storia della Chiesa - relatore il Professor Umberto Mazzone, correlatore il Professor Enrico Morini - dal titolo 
Epiro crocifisso o liberato? La Chiesa ortodossa in Epiro e in Albania meridionale nel XX secolo (1912-1967). Ha lavorato come redattore per la casa editrice L'Inventaire e ha curato il progetto Dictionnaire universel des femmes créatrices - secteur "Femmes du livre" in corso di pubblicazione presso Editions des Femmes. Attualmente collabora con la casa editrice Il Mulino alla creazione dell'archivio digitale dei libri del Mulino, Darwinbooks e con la casa editrice Carocci.

\section{LUCA BUFARALE}

Ha conseguito nel 2008 la laurea specialistica in storia d'Europa presso l'Università di Bologna e nel 2012 il dottorato di ricerca in scienze storiche (indirizzo contemporaneo) presso l'Università di Padova con una tesi dal titolo La giovinezza politica di Riccardo Lombardi (1919-1949). Ha condotto i suoi primi studi sull'Unione Sovietica pubblicando il saggio «Per un socialismo di mercato. Aspetti del dibattito economico in URSS negli anni sessanta» (in Storicamente, 2, 006, http://www.storicamente.org/05_studi_ricerche/02bufarale.htm); successivamente ha indirizzato le sue ricerche sulla figura politica e intellettuale del leader azionista e poi socialista Riccardo Lombardi. È membro della Società italiana per lo studio della storia contemporanea e dall'Association for the study of Modern Italy. Su Lombardi ha pubblicato articoli su «Il Ponte», sugli «Annali della Fondazione Ugo La Malfa» e su «Diacronie. Studi di Storia Contemporanea». Ha partecipato come relatore ad un convegno promosso dall'Associazione nazionale Riccardo Lombardi (Torino, 7 novembre 2009) e alla sesta edizione di "Giellismo e azionismo: cantieri aperti" (Torino, 6 - 8 maggio 2010). Su Lombardi ha pubblicato un articolo su «Il Ponte» (marzo 2010). È di prossima pubblicazione un suo saggio negli «Annali della Fondazione Ugo La Malfa».

\section{MATTEO TOMASONI}

Laureato nel 2005 presso l'Università di Trieste in Storia contemporanea (indirizzo informatico) con una tesi intitolata Il ruolo di Valladolid nella Spagna contemporanea, relatore il prof. Sergio Zilli. Nel 2004 partecipa ad un programma di scambio Socrates/Erasmus presso l'Universidad de Valladolid (Spagna), da cui nasce una collaborazione con il Dipartimento di Historia Moderna, Contemporánea y de América e quello di Geografía. Nel 2008 consegue il titolo di Laurea Specialistica (Master 2) in Storia d'Europa presso l' Università di Bologna, con una tesi di laurea intitolata: Opinione pubblica e fascismo spagnolo. La Valladolid di Onésimo Redondo, sotto la direzione del Prof. Luciano Casali. Attualmente si è trasferito in Spagna dove ha iniziato il Dottorato di ricerca ("Cambios y comunicación en la historia") presso l'Università di Valladolid. Si occupa di studi relativi alla Spagna contemporanea, soprattutto di carattere socio-politico, interessandosi inoltre a tematiche specifiche dell'area Castigliana, attraverso l'analisi della nascita dei primi movimenti legati al falangismo. 